\title{
Bourbaki and Palais Proper Actions on $d$-Algebra
}

\author{
Ahmed Talip Hussein ${ }^{1}$, Habeeb Kareem Abdulla ${ }^{2}$ and Haider Jebur Ali $^{3}$ \\ ${ }^{1}$ Department of Mathematics, Faculty of Computer Science and Information Technology, \\ University of Al-Qadisiyah, Al-Qadisiyah, Iraq; e-mail: Ahmed.talip@ qi.edu.iq \\ ${ }^{2}$ Department of Mathematics, Faculty of Education for Girls, University of Kufa, Najef, Iraq \\ e-mail: Habeebk.abdullah@uokufa.edu.iq \\ ${ }^{3}$ Department of Mathematics, College of Science, Al-Mustansiriyah University, Baghdad, Iraq \\ e-mail: Haiderali@yahoo.com
}

\begin{abstract}
The main goal of this paper is to create a general two types of proper $D$-space, namely, Bourbaki proper $D$-space and Palais proper $D$-space, to explain the relation between Bourbaki proper and Palais proper $D$-spaces and to study some of examples and propositions of Bourbaki proper and Palais proper $D$-spaces.
\end{abstract}

\section{Introduction}

One of the very important concepts in topological $d$-algebras is the concept of $d$-algebra actions. This paper studies an important class of actions namely, Bourbaki proper $D$-spaces.

Imai and Iséki [6] and Iséki [7] introduced two classes of abstract algebras: namely, BCK-algebras and BCI-algebras. It is known that the class of BCK algebras is a proper subclass of the class of BCI-algebras. In [4], [5] Hu and Li introduced a wide class of abstract algebras: $\mathrm{BCH}$-algebras. They have shown that the class of BCI-algebras is a proper subclass of the class of BCH-algebras. Neggers et al. [8] introduced the notion of Received: March 7, 2019; Revised \& Accepted: June 27, 2019

2010 Mathematics Subject Classification: 06F35.

Keywords and phrases: $d$-algebra, $d$-ideal, topological $d$-algebras, Bourbaki proper $D$-space, Palais proper $D$-space.

Copyright (C) 2019 Ahmed Talip Hussein, Habeeb Kareem Abdulla and Haider Jebur Ali. This is an open access article distributed under the Creative Commons Attribution License, which permits unrestricted use, distribution, and reproduction in any medium, provided the original work is properly cited. 
$d$-algebras which is another generalization of BCK-algebras, and investigated relations between $d$-algebras and BCK-algebras. They studied the various topologies in a manner analogous to the study of lattices. However, no attempts have been made to study the topological structures making the star operation of $d$-algebra continuous. Theories of topological groups, topological rings and topological modules are well known and still investigated by many mathematicians. Even topological universal algebraic structures have been studied by some authors.

In Section 1, we include some of results which will be needed in the next sections. In Section 2, we deal with the definitions, examples, remarks, propositions, theorem and corollaries of proper function. In Section 3, we deal with the definitions, examples, remarks and propositions of topological $d$-algebra. We also provided in this section the definition of Bourbaki proper $D$-space, gives a new type of Bourbaki proper $D$-space (to the best of our knowledge), namely, proper $D$-space and studies some of its properties, where $D$-space is meant $T_{2}$-space topological $X$ on which a locally compact, noncompact, $T_{2}$-topological $d$-algebra $D$ acts continuously on the left. In Section 4 , the definitions, propositions, theorems and examples of a Palais proper $D$-space are given as well as the relation between Bourbaki proper and Palais proper $D$-spaces is studied.

\section{Preliminaries}

Definition 2.1 [1]. Let $X$ and $Y$ be spaces and $f: X \rightarrow Y$ be a function. Then $f$ is called continuous function if $f^{-1}(A)$ is an open set in $X$ for every open set $A$ in $Y$.

Proposition 2.2 [1]. Let $f: X \rightarrow Y$ be a function of spaces. Then $f$ is a continuous function if and only if $f^{-1}(A)$ is a closed set in $X$ for every closed set $A$ in $Y$.

Definition 2.3 [1]. (i) A function $f: X \rightarrow Y$ is called closed function if the image of each closed subset of $X$ is a closed set in $Y$.

(ii) A function $f: X \rightarrow Y$ is called open function if the image of each open subset of $X$ is an open set in $Y$.

Definition 2.4 [1]. Let $X$ and $Y$ be spaces. Then a function $f: X \rightarrow Y$ is called a homeomorphism if:

(i) $f$ is bijective. 
(ii) $f$ is continuous.

(iii) $f$ is closed (open).

Definition 2.5 [1]. Let $\left(\chi_{g}\right)_{g \in G}$ be a net in a space $X, x \in X$. Then:

(i) $\left(\chi_{g}\right)_{g \in G}$ converges to $x$ (written $\chi_{g} \rightarrow x$ ) if $\left(\chi_{g}\right)_{g \in G}$ is eventually in every neighborhood of $x$. The point $x$ is called a limit point of $\left(\chi_{g}\right)_{g \in G}$, and the notation " $\chi_{g} \rightarrow \infty$ " is mean that $\left(\chi_{g}\right)_{g \in G}$ has no convergent subnet.

(ii) $\left(\chi_{g}\right)_{g \in G}$ is said to have $x$ as an cluster point [written $\chi_{g} \propto x$ ] if $\left(\chi_{g}\right)_{g \in G}$ is frequently in every neighborhood of $x$.

Proposition 2.6 [1]. Let $\left(\chi_{g}\right)_{g \in G}$ be a net in a space $(X, T)$ and $x_{0}$ in $X$. Then $\chi_{g} \propto x_{0}$ if and only if there exists a subnet of $\left(\chi_{g_{m}}\right)_{g_{m} \in G}$ of $\left(\chi_{g}\right)_{g \in G}$ such that $\chi_{g_{m}} \rightarrow x_{0}$.

Remark 2.7 [1]. Let $\left(\chi_{g}\right)_{g \in G}$ be a net in a space $(X, T)$ such that $\chi_{g} \propto x, x \in X$ and let $A$ be an open set in $X$ which contains $x$. Then there exists a subnet $\left(\chi_{g_{m}}\right)_{g_{m} \in G}$ of $\left(\chi_{g}\right)_{g \in G}$ in the set $A$ such that $\chi_{d_{m}} \rightarrow x$.

Definition 2.8 [1]. A subset $A$ of space $X$ is called compact set if every open cover of $A$ has a finite sub cover. If $A=X$, then $X$ is called a compact space.

Proposition 2.9 [1]. Let $X$ be a space and $F$ be a closed subset of $X$. Then $F \cap K$ is compact subset of $F$, for every compact set $K$ in $X$.

Proposition 2.10 [1]. Let $Y$ be an open subspace of space $X$ and $A \subseteq Y$. Then $A$ is an compact set in $Y$ if and only if $A$ is an compact set in $X$.

Definition 2.11 [1]. (i) A subset $A$ of space $X$ is called relative compact if $\bar{A}$ is compact.

(ii) A space $X$ is called locally compact if every point in $X$ has a relative compact neighborhood.

Proposition 2.12 [1]. Let $X$ and $Y$ be spaces and $f: X \rightarrow Y$ be a continuous function. Then an image $f(A)$ is compact in $Y$ for every $A$ is compact in $X$. 
Definition 2.13 [1]. Let $f: X \rightarrow Y$ be a function of spaces. Then $f$ is called a compact function if $f^{-1}(A)$ is a compact set in $X$ for every compact set $A$ in $Y$.

Proposition 2.14 [1]. Let $X, Y$ be spaces and $f: X \rightarrow Y$ be compact function. If $F$ is a closed subset of $X$ and $B$ is an open set in $Y$, then $f_{/ F}: F \rightarrow B$ is compact.

Definition 2.15 [1]. Let $X$ and $Y$ be two spaces. Then $f: X \rightarrow Y$ is called a proper function if:

(i) $f$ is continuous function.

(ii) $f \times I_{Z}: X \times Z \rightarrow Y \times Z$ is a closed function, for every space $Z$.

Proposition 2.16 [1]. Let $X$ and $Y$ be spaces and $f: X \rightarrow Y$ be a continuous function. Then the following statements are equivalent:

(i) $f$ is a proper function.

(ii) $f$ is a closed function and $f^{-1}(\{y\})$ is an compact set, for each $y \in Y$.

(iii) If $\left(\chi_{g}\right)_{g \in G}$ is a net in $X$ and $y \in Y$ is a cluster point of $f\left(\chi_{g}\right)$, then there is a cluster point $x \in X$ of $\left(\chi_{g}\right)_{g \in G}$ such that $f(x)=y$.

Proposition 2.17 [1]. Let $X, Y$ and $Z$ be spaces, $f: X \rightarrow Y$ and $g: Y \rightarrow Z$ be two proper functions. Then $g$ of $: X \rightarrow Z$ is a proper function.

Proposition 2.18 [1]. Let $f_{1}: X_{1} \rightarrow Y_{1}$ and $f_{2}: X_{2} \rightarrow Y_{2}$ be two functions. Then $f_{1} \times f_{2}: X_{1} \times X_{2} \rightarrow Y_{1} \times Y_{2}$ is proper function if and only if $f_{1}$ and $f_{2}$ are proper functions.

Proposition 2.19 [1]. Every proper function is closed.

Proposition 2.20 [1]. Let $f: X \rightarrow P=\{w\}$ be a continuous function on a space $X$. Then $f$ is a proper function if and only if $X$ is a compact, where $w$ is any point which does not belongs to $X$.

Lemma 2.21 [1]. Every continuous function from a compact space into a Hausdorff space is closed. 
Remark 2.22 [1]. If $X$ is a space, then the diagonal function $\Delta: X \rightarrow X \times X$ such that $\Delta(x)=(x, x)$ is continuous.

Proposition 2.23. If $X$ is a Hausdorff space, then the diagonal function $\Delta: X \rightarrow X \times X$ is proper function.

Proof. Let $\left(\chi_{g}, \chi_{g}\right) \propto\left(x_{1}, x_{2}\right)$. So there exists a subnet of $\left(\chi_{g}, \chi_{g}\right)$, say itself, such that $\left(\chi_{g}, \chi_{g}\right) \rightarrow\left(x_{1}, x_{2}\right)$, then $\chi_{g} \rightarrow x_{1}$ and $\chi_{g} \rightarrow x_{2}$. Since $X$ is a $T_{2}$-space, $x_{1}=x_{2}$. Then there is $x_{1} \in X$ such that $\chi_{g} \propto x_{1}$ and $\Delta\left(x_{1}\right)=y$. Hence by Proposition 2.16(iii), $\Delta$ is a proper function.

Proposition 2.24 [1]. Let $f_{1}: X \rightarrow Y_{1}$ and $f_{2}: X \rightarrow Y_{2}$ be two proper functions. If $X$ is a Hausdorff space, then the function $f_{1}: X \rightarrow Y_{1} \times Y_{2}, f(x)=\left(f_{1}(x), f_{2}(x)\right)$ is a proper function.

Proposition 2.25 [1]. Let $X$ and $Y$ be spaces and $f: X \rightarrow Y$ be a continuous, one to one function. Then the following statements are equivalent:

(i) $f$ is a proper function.

(ii) $f$ is a closed function.

(iii) $f$ is a homeomorphism of $X$ onto closed subset of $Y$.

Proposition 2.26 [1]. Let $X$ and $Y$ be spaces such that $Y$ is a $T_{2}$-space and $f: X \rightarrow Y$ be a continuous function. Then the following statements are equivalent:

(i) $f$ is a compact function.

(ii) $f$ is a proper function.

(iii) If $\left(\chi_{g}\right)_{g \in G}$ is a net in $X$ and $y \in Y$ is a cluster point of $f\left(\chi_{g}\right)$, then there is a cluster point $x \in X$ of $\left(\chi_{g}\right)_{g \in G}$ such that $f(x)=y$.

\section{Bourbaki Proper D-Space}

In this section, we examine the definition of topological $d$-algebra and some issues and examples related to the subject and we define the space Bourbaki proper $D$-space.

Definition 3.1 [8]. A non-empty set $D$ together with a binary operation $*$ and a zero element 0 is said to be a $d$-algebra if the following axioms are satisfied for all $x, y \in D$ 
(1) $x * x=0$

(2) $0 * x=0$

(3) $x * y=0$ and $y * x=0$ imply that $x=y$.

Definition 3.2 [2]. An element $e$ of $D$ is called a left identity if $e * a=a$, a right identity if $a * e=a$ for all $a \in D$ and $a \neq e$. If $e$ is both left and right identity, then we called $e$ is an identity element. Also we say that $(D, *)$ is $d$-algebra with identity element.

Example 3.3. (i) Let $D$ be any non-empty set and $P(D)$ be power set of $D$. Then $(P(D),-)$ is $d$-algebra and $\phi$ is right identity in $(P(D),-)$.

(ii) Let $D=\{0, a, b, c\}$ and define the binary operation $*$ on $D$ by following table:

\begin{tabular}{|l|l|l|l|l|}
\hline$*$ & 0 & $a$ & $b$ & $c$ \\
\hline 0 & 0 & 0 & 0 & 0 \\
\hline$a$ & 0 & 0 & $b$ & $c$ \\
\hline$b$ & 0 & $b$ & 0 & $a$ \\
\hline$c$ & 0 & $c$ & $a$ & 0 \\
\hline
\end{tabular}

Table 1

Then the pair $(D, *)$ is $d$-algebra with identity element $a$.

Definition 3.4 [2]. Let $(D, *)$ be a $d$-algebra and $T$ be a topology on $D$. Then the triple $(D, *, T)$ is called a topological d-algebra (denoted by $T d$-algebra) if the binary operation $*$ is continuous.

Example 3.5. (i) Let $D=\{0, a, b, c\}$ and $*$ be defined by following table:

\begin{tabular}{|l|l|l|l|l|}
\hline$*$ & 0 & $a$ & $b$ & $c$ \\
\hline 0 & 0 & 0 & 0 & 0 \\
\hline$a$ & $a$ & 0 & 0 & $a$ \\
\hline$b$ & $b$ & $b$ & 0 & $b$ \\
\hline$c$ & $c$ & $c$ & $c$ & 0 \\
\hline
\end{tabular}

Table 2 
It is clear that $(D, *)$ is $d$-algebra and $T=\{\varnothing,\{b\},\{c\},\{0, a\},\{b, c\},\{0, a, b\}$, $\{0, a, c\}, D\}$ is topology on $D$ such that the triple $(D, *, T)$ is a topological $d$-algebra.

(ii) Let $R$ be a set of real numbers and $*$ be a binary operation defined by $a * b=a \cdot(a-b)^{2}$. Then $(R, *)$ is $d$-algebra and $(R, *, T)$ is $T d$-algebra, where $T$ is usual topology on $R$.

Definition 3.6 [2]. A topological transformation $d$-algebra is a triple $(D, X, \varphi)$, where $D$ is a $T_{2}$-topological $d$-algebra with left identity $e, X$ is a $T_{2}$-topological space and $\varphi: D \times X \rightarrow X$ is a continuous function such that:

(i) $\varphi\left(d_{1}, \varphi\left(d_{2}, x\right)\right)=\varphi\left(d_{1} d_{2}, x\right)$ for all $d_{1} \neq d_{2}$ and $d_{1}, d_{2} \in D, x \in X$.

(ii) $\varphi(e, x)=x$ for all $x \in X$, where $e$ is the left identity element of $D$.

We shall often use the notation $d \cdot x$ for $\varphi(d, x)$ and $d \cdot(h, x)=(d h) \cdot x$ for $\varphi(d, \varphi(h, x))=\varphi(d h, x)$. Similarly for $H \subseteq D$ and $A \subseteq X$ we put $H A=$ $\{h a / h \in H, a \in A\}$ for $\varphi(H, A)$.

Remark 3.7 [2]. (i) The function $\varphi$ is called an action of $D$ on $X$ and the space $X$ together with $\varphi$ is called a $D$-space (or more precisely left $D$-space ).

(ii) The subspace $\{d \cdot x / d \in D\}$ is called the orbit (trajectory) of $x$ under $D$, which denoted by $D x$ [or $\gamma(x)$ ], and for every $x \in X$ the stabilizer subgroup $D_{x}$ of $D$ at $x$ is the set $\{d \in D / d \cdot x=x\}$.

(ii) A set $A \subseteq X$ is said to be invariant under $D$ if $D A=A$.

Definition 3.8. A $D$-space $X$ is called a Bourbaki proper D-space (proper D-space) if the function $\theta: D \times X \rightarrow X \times X$ defined by $\theta(d, x)=(x, d \cdot x)$ is a proper function.

Example 3.9. Let $Z_{3}=\{-1,0,1\}$ and $*$ be defined by following table:

\begin{tabular}{|c|c|c|c|}
\hline$*$ & 0 & -1 & 1 \\
\hline 0 & 0 & 0 & 0 \\
\hline-1 & 0 & 0 & -1 \\
\hline 1 & 0 & -1 & 0 \\
\hline
\end{tabular}

Table 3 
Then $\left(Z_{3}, *\right)$ is $d$-algebra and $\left(Z_{3}, *, \tau\right)$ is $T d$-algebra, where $\tau$ is discrete topology on $Z_{3}$. The act on the topological space $S^{n}$ [as a subspace of $R^{n+1}$ with usual topology] as follows:

$$
\begin{gathered}
0 \cdot\left(x_{1}, x_{2}, \ldots, x_{n+1}\right)=(0,0, \ldots, 0) \\
1 \cdot\left(x_{1}, x_{2}, \ldots, x_{n+1}\right)=\left(x_{1}, x_{2}, \ldots, x_{n+1}\right) \\
-1 \cdot\left(x_{1}, x_{2}, \ldots, x_{n+1}\right)=\left(-x_{1},-x_{2}, \ldots,-x_{n+1}\right) .
\end{gathered}
$$

Since $Z_{3}$ is compact, by Proposition 2.20, the constant function $Z_{3} \rightarrow P$ is a proper. Also the identity function is a proper, then by Proposition 2.18, the proper function of $Z_{3} \times S^{n}$ into $P \times S^{n}$ is a proper.

Since $P \times S^{n}$ is homeomorphic to $S^{n}$, by Proposition 2.25, the homeomorphism of $P \times S^{n}$ onto $S^{n}$ is proper. Since $Z_{3} \times S^{n} \rightarrow P \times S^{n}$ is continuous and open function, by Proposition 2.17, the composition $Z_{3} \times S^{n} \rightarrow S^{n}$ is a proper. Let $\varphi$ be the action of $Z_{3}$ on $S^{n}$. Then $\varphi$ is continuous, one to one function, so $\varphi$ is continuous function. Since $S^{n}$ is $T_{2}$-space, by Lemma 2.21, $\varphi$ is a closed. Then by Proposition 2.25, $\varphi$ is a proper function. Thus by Proposition 2.24, $Z_{3} \times S^{n} \rightarrow S^{n} \times S^{n}$ is a proper $D$-space.

Lemma 3.10. If $X$ is a $D$-space, then the function $\theta: D \times X \rightarrow X \times X$ defined by $\theta(d, x)=(x, d \cdot x)$ is a continuous function and $\theta^{-1}(\{(x, y)\})$ is closed in $D \times X$ for every $(x, y) \in X \times X$.

Proof. Since

$\theta: D \times X \stackrel{I_{D} \times \Delta}{\longrightarrow} D \times X \times X \stackrel{\varphi \times I_{X}}{\longrightarrow} X \times X \stackrel{f}{\stackrel{f}{\cong}} \longrightarrow \times X$,

where $\varphi$ is action of $D$ on $X$. Then $\theta=f \circ \varphi \times I_{X} \circ I_{D} \times \Delta$ is continuous function and $\theta^{-1}(\{(x, y)\})$ is closed in $D \times X$ for every $(x, y) \in X \times X$.

Theorem 3.11. Let $X$ be a proper D-space and let $H$ be a closed subset of D. If $Y$ is an open subset of $X$ which is invariant under $H$, then $Y$ is a proper $H$-space.

Proof. Since $X$ is a proper $D$-space, the function $\theta: D \times X \rightarrow X \times X$ defined by 
$\theta(d, x)=(x, d \cdot x)$ is a proper function. [To prove that $\omega: H \times Y \rightarrow Y \times Y$ is a proper function defined by $\omega(h, y)=\theta(h, y)$ for each $(h, y) \in H \times Y]$.

(1) By Lemma 3.10, $\theta: D \times X \rightarrow X \times X$ is continuous, then $\omega: H \times Y \rightarrow Y \times Y$ is continuous.

(2) Let $\left(h_{g}, y_{g}\right)_{g \in G}$ be a net in $H \times Y$ such that $\omega\left(\left(h_{g}, y_{g}\right)\right) \propto(x, y)$ for some $(x, y) \in Y \times Y$. Then $\left(y_{g}, h_{g} y_{g}\right) \propto(x, y)$ in $Y \times Y$. Let $A$ be an open subset of $X \times X$ such that $(x, y) \in A$. Since $Y$ is open in $X, Y \times Y$ is an open set in $X \times X$. Then $A \cap(Y \times Y)$ is an open set in $X \times X$. But $(x, y) \in A \cap(Y \times Y)$ and $\left(y_{g}, h_{g} y_{g}\right) \propto$ $(x, y)$, thus $\left(y_{g}, h_{g} y_{g}\right)$ is frequently in $A \cap(Y \times Y)$ and then $\left(y_{g}, h_{g} y_{g}\right)$ is frequently in $A$, thus $\left(y_{g}, h_{g} y_{g}\right) \propto(x, y)$ in $X \times X$. Since $\theta: D \times X \rightarrow X \times X$ is an proper function, by Proposition 2.16, there exists $\left(h, x_{1}\right) \in D \times X$ such that $\left(h_{g}, y_{g}\right) \propto\left(h, x_{1}\right)$ and $\theta\left(\left(h, x_{1}\right)\right)=(x, y)$. Hence $\left(x_{1}, h x_{1}\right)=(x, y)$. Thus $x_{1}=x$ and therefore $h_{g} \propto h$. Since $\left(h_{g}\right)_{g \in G}$ is a net in $H$, and $H$ is closed. Then there exists $(h, x) \in H \times Y$ such that $\omega(h, x)=\theta(h, x)=(x, y)$. Then from (1), (2) and by Proposition 2.16, the function $\omega: H \times Y \rightarrow Y \times Y$ is a proper function. Hence $Y$ is a proper $H$-space.

Corollary 3.12. Let $X$ be a proper D-space and $Y$ be an open subset of $X$ which is invariant under $D$. Then $Y$ is a proper D-space.

Corollary 3.13. Let $X$ be a proper D-space and let $H$ be closed subset of D. Then $X$ is a proper H-space.

Proposition 3.14. Let $X$ be a proper D-space, $x \in X$ and $T=\{x\} \times X$. Then the function $\theta_{T}: \theta^{-1}(T) \rightarrow T$ is a proper function, where $\theta: D \times X \rightarrow X \times X$ such that $\theta(g, x)=(x, g \cdot x), \forall(g, x) \in D \times X$.

Proof. Since $X$ is a $T_{2}$-space, $\{x\}$ is closed set in $X$. Thus, $\{x\}$ is closed set in $X$. So each $D \times\{x\}$ and $\{x\} \times X$ are closed in $D \times X$ and $X \times X$ (respectively). Now, let $F$ be a closed set in $\theta^{-1}(T)=D \times\{x\}$. Since $F=F \cap(D \times\{x\}), F$ is closed in $D \times X$ and $\theta_{T}(F)=\theta(F) \cap(\{x\} \times X)$. Therefore $\theta(F)$ is closed in $X \times X$, then by 
Proposition 2.19, $\theta_{T}(F)$ is closed in $X \times X$. But $\theta_{T}(F) \subseteq\{x\} \times X$, then there exists a subset $V$ of $X$ such that $\theta_{T}(F)=\{x\} \times V$. Since $\theta_{T}(F)$ is closed in $X \times X$, so $\{x\} \times V$ is a closed set in $\{x\} \times X$. Hence $\theta_{T}(F)=\{x\} \times V$ is a closed set in $T=\{x\} \times X$, therefore $\theta_{T}: \theta^{-1}(T) \rightarrow T$ is closed. Now, let $(x, y) \in\{x\} \times X$. Since $\theta$ is proper function, by Proposition 2.26, $\theta$ is a compact function. Then $\theta^{-1}(\{(x, y)\})$ is compact in $D \times X$. Then by $\theta_{T}^{-1}(\{(x, y)\})$ is compact set in $D \times\{x\}=\theta^{-1}(T)$. Thus by Proposition $2.26, \theta_{T}$ is a proper function.

Let $X$ be a $D$-space and $A, B$ be two subsets of $X$. We mean by $((A, B))$ the set $\{d \in D / d A \cap B \neq \varnothing\}$.

From now on, we will use $D$-space, which satisfies the property if $(X, T)$ and $\left(Y, T^{\prime}\right)$ be two space and $\forall \chi_{g} \rightarrow x, y_{g} \rightarrow y$ in $X$ and $Y$, respectively, then $\left(\chi_{g}, y_{g}\right) \rightarrow(x, y)$.

Proposition 3.15. Let $X$ be a $D$-space. If for every $x, y \in X$ there exist an open set $A_{x}$ of $X$ contains $x$ and an open set $A_{y}$ of $X$ contains $y$ such that $K=\left(\left(A_{x}, A_{y}\right)\right)$ is relatively compact in $D$, then $X$ is a proper $D$-space.

Proof. We prove that $\theta: D \times X \rightarrow X \times X, \theta(d, x)=(x, d x)$ is a proper function. Let $\left(d_{g}, \chi_{g}\right)_{g \in G}$ be a net in $D \times X$ such that $\theta\left(\left(d_{g}, \chi_{g}\right)\right)=\left(\chi_{g}, d_{g} \chi_{g}\right) \propto(x, y)$, where $(x, y) \in X \times X$. Now, since $x, y \in X$, there exist an open set $A_{x}$ contains $x$ and an open set $A_{y}$ contains $y$ such that the set $K=\left(\left(A_{x}, A_{y}\right)\right)$ is relatively compact in $D$. Thus, $A_{x} \times A_{y}$ is an open set in $X \times X$ and $(x, y) \in A_{x} \times A_{y}$. So there exists a subnet $\left(\chi_{g_{m}}, d_{g_{m}} \chi_{g_{m}}\right)_{g \in G}$ of $\left(\chi_{g}, d_{g} \chi_{g}\right)$ in $A_{x} \times A_{y}$ and $\left(\chi_{g_{m}}, d_{g_{m}} \chi_{g_{m}}\right) \rightarrow(x, y)$, hence $\chi_{g_{m}} \rightarrow x \quad$ and $\quad d_{g_{m}} \chi_{g_{m}} \rightarrow y$. Since $\chi_{g_{m}} \in A_{x} \quad$ and $\quad d_{g_{m}} \chi_{g_{m}} \in A_{y}$. Then $d_{g_{m}} A_{x} \cap A_{y} \neq \varnothing, \forall g_{m}$, so $d_{g_{m}} \in K$, but $K$ is relatively compact in $D$, then by Proposition 2.6, $\left(d_{g_{m}}\right)$ has a limit point, say $t \in D$. Since $\chi_{g_{m}} \rightarrow x$, then $\left(d_{g}, \chi_{g}\right) \rightarrow(t, x)$, so $\theta\left(\left(d_{g}, \chi_{g}\right)\right) \rightarrow \theta((t, x))$, i.e., $\left(\chi_{g_{m}}, d_{g_{m}} \chi_{g_{m}}\right) \rightarrow(x, t x)$, thus $d_{g_{m}} \chi_{g_{m}} \rightarrow t x$ but $d_{g_{m}} \chi_{g_{m}} \rightarrow y$ and since $X$ is a Hausdorff space, then $t x=y$. But 
$\left(\chi_{g_{m}}, g_{g_{m}} \chi_{g_{m}}\right)_{g \in G}$ is a subnet of $\left(\chi_{g}, d_{g} \chi_{g}\right)$ and $\left(d_{g_{m}}, \chi_{g_{m}}\right) \rightarrow(t, x)$, then $\left(d_{g}, \chi_{g}\right) \propto(t, x)$, thus $\theta((t, x))=(x, y)$. Then by Proposition 2.16, we have $\theta$ is a proper function. Hence $X$ is a proper $D$-space.

Corollary 3.16. Let $X$ be a $D$-space such that $D$ is discrete space. If for every $x, y \in X$ there is an open set $A_{x}$ in $X$ contains $x$ and an open set $A_{y}$ in $X$ contains $y$ such that the set $K=\left(\left(A_{x}, A_{y}\right)\right)$ is finite, then $X$ is a proper $D$-space.

Let $X$ be a $D$-space and $x \in X$. The set $J(x)=\left\{y \in X\right.$ : there is a net $\left(d_{g}\right)_{g \in G}$ in $D$ and there is a net $\left(\chi_{g}\right)_{g \in G}$ in $X$ with $d_{g} \rightarrow \infty$ and $\chi_{g} \rightarrow x$ such that $\left.d_{g} x \rightarrow y\right\}$ is called first prolongation limit set of $x$, [3]. $J(x)$ is a good tool to discover about the Bourbaki proper $D$-space.

Proposition 3.17. Let $X$ be a D-space. Then $X$ is a Bourbaki proper D-space if and only if $J(x)=\varnothing$ for each $x \in X$.

Proof. $\Rightarrow$ Suppose that $y \in J(x)$, then there is a net $\left(d_{g}\right)_{g \in G}$ in $D$ with $d_{g} \rightarrow \infty$ and there is a net $\left(\chi_{g}\right)_{g \in G}$ in $X$ with $\chi_{g} \rightarrow x$ such that $d_{g} \chi_{g} \rightarrow y$, so $\theta\left(\left(d_{g}, \chi_{g}\right)\right)$ $=\left(\chi_{g}, d_{g} \chi_{g}\right) \rightarrow(x, y)$. But $X$ is a Bourbaki proper, then by Proposition 2.26, there is $\left(d, x_{1}\right) \in d \times X$ such that $\left(d_{g}, \chi_{g}\right) \propto\left(d, x_{1}\right)$. Thus, $\left(d_{g}\right)_{g \in G}$ has a subnet (say itself), such that $d_{g} \rightarrow d$, which is contradiction, thus $J(x)=\varnothing$.

$\Leftarrow$ Let $\left(d_{g}, \chi_{g}\right)_{g \in G}$ be a net in $D \times X$ and $(x, y) \in X \times X$ such that $\theta\left(\left(d_{g}, \chi_{g}\right)\right)=\left(\chi_{g}, d_{g} \chi_{g}\right) \propto(x, y)$ so $\left(\chi_{g}, d_{g} \chi_{g}\right)_{g \in G}$ has a subnet, say itself, such that $\left(\chi_{g}, d_{g} \chi_{g}\right) \rightarrow(x, y)$, then $\chi_{g} \rightarrow x$ and $d_{g} \chi_{g} \rightarrow y$. Suppose that $d_{g} \rightarrow \infty$, then $y \in J(x)$, which is contradiction. Then there is $d \in D$ such that $d_{g} \rightarrow d$, then $\left(d_{g}, \chi_{g}\right) \rightarrow(d, x)$ and $\theta(d, x)=(x, y)$. Thus by Lemma 3.10 and Proposition 2.16, $X$ is a Bourbaki proper $D$-space.

Proposition 3.18. Let $X$ be a proper $D$-space, $\theta(d, x)=(x, d \cdot x) \forall(d, x) \in D \times X$ with the action $\varphi: D \times X \rightarrow X, \varphi(d, x)=d \cdot x, \forall(d, x) \in D \times X$. Then for each 
$x \in X$, the function $\varphi_{x}: D \rightarrow X$, which is defined by: $\varphi_{x}(d)=\varphi(d, x)$ is a proper function.

Proof. Let $T=\{x\} \times X \subseteq X \times X$. Then by Proposition 3.14, $\theta_{T}: \theta^{-1}(T) \rightarrow T$ is a proper function. But:

$$
\varphi_{x}=D \stackrel{f}{\cong} D \times\{x\} \stackrel{\theta_{T}}{\longrightarrow}\{x\} \times X \stackrel{h}{\cong} X \text {, such that } f \text { and } h \text { are homeomorphisms. }
$$

Now:

(i) Let $A$ be an open set in $X$. Then $h^{-1}(A)$ is an open set in $\{x\} \times X$. Since $\theta_{T}$ is continuous, $\theta_{T}^{-1}\left(h^{-1}(A)\right)$ is open in $D \times\{x\}$. Since $f$ is homeomorphism, $f^{-1}\left(\theta_{T}^{-1}\left(h^{-1}(A)\right)\right)$ is open in $D$. Then $\varphi_{x}(A)=f^{-1} \circ \theta_{T}^{-1} \mathrm{o} h^{-1}(A)$ is open in $D$. Thus, $\varphi_{x}: D \rightarrow X$ is continuous.

(ii) Let $F$ is closed in $D$. Then $h(F)$ is closed in $\{x\} \times X$. Since $\theta_{T}: D \times\{x\}$ $\rightarrow\{x\} \times X$ is a proper function, by Proposition 2.19, $\theta_{T}(h(F))$ is closed. Then $\varphi_{x}(F)=f\left(\theta_{T}(h(F))\right)$ is closed in $X$. Then $\varphi_{x}: D \rightarrow X$ is closed.

(iii) Let $y \in X$. Then $h^{-1}(\{y\})=\{(x, y)\}$ such that $x \in X$. Since $X$ is $T_{2}$-space, $\{(x, y)\}$ is a closed set in $\{x\} \times X$. Since $\theta_{T}$ is a continuous function, $\theta_{T}^{-1}(\{(x, y)\})$ is closed in $D \times\{x\}$. So by Proposition 2.16, $\theta_{T}^{-1}\left(h^{-1}(\{y\})\right)=\theta_{T}^{-1}\{(x, y)\}$ is compact. Since $f$ is homeomorphism, it is clear that $f^{-1}\left(\theta_{T}^{-1}\{(x, y)\}\right)$ is compact in $D$. Then $f^{-1}\left(\theta_{T}^{-1}\left(h^{-1}(\{y\})\right)\right)=\varphi_{x}^{-1}(\{y\})$ is compact in $D$. Then by (i), (ii), (iii) and Proposition $2.16, \varphi_{x}$ is proper function.

Proposition 3.19. Let $X$ be a $D$-space and $\theta: D \times X \rightarrow X \times X$ be a function defined by $\theta(d, x)=(x, d x), \forall(d, x) \in D \times X$. Then the following statements are equivalent:

(i) $X$ is a proper D-space.

(ii) $\theta^{-1}(\{(x, y)\})$ is a compact set, $\forall(x, y) \in X \times X$ and for all $x, y \in X$ and for all $U \in N(((x, y))), \exists V_{x} \in N(x)$ and $V_{y} \in N(y)$ such that $\left(\left(V_{x}, V_{y}\right)\right) \subseteq U$. 
(iii) $\theta^{-1}(\{(x, y)\})$ is a compact set, $\forall(x, y) \in X \times X$ and for all $x, y \in X$ and for all $U \in N\left(\theta^{-1}(\{(x, y)\})\right), \exists V \in N((x, y))$ such that $\theta^{-1}(V) \subseteq U$.

Proof. (i) $\rightarrow$ (iii) Let $x, y \in X$ and $U$ be an open neighborhood of $\theta^{-1}(x, y)$. Since $\theta$ is a proper function, by Proposition 2.19, $\theta$ is a closed function. So $V=(X \times X) \backslash \theta((D \times X) \backslash U)$ is an open neighborhood of $(x, y)$ with $\theta^{-1}(V) \subseteq U$. So by Proposition 2.16, $\theta^{-1}(\{(x, y)\})$ is a compact set $\forall(x, y) \in X \times X$. Hence (iii) holds.

(iii) $\rightarrow$ (i) Let $F$ be a closed subset of $D \times X$ and let $(x, y) \in X \times X \backslash \theta(F)$. Since $(D \times X) \backslash F$ is an open neighborhood of $\theta^{-1}(x, y)$, by (iii) there is a neighborhood $V$ of $(x, y)$ such that $\theta^{-1}(V) \subseteq(D \times X) \backslash F$. Hence $V \cap \theta(F)=\varnothing$, so $(x, y) \notin \overline{\theta(F)}$, then $\overline{\theta(F)}=\theta(F)$. Hence $\theta$ is a closed function. Since $\theta^{-1}(\{(x, y)\})$ is a compact set for every $(x, y) \in X \times X$, by Proposition $2.16, \theta$ is a proper function. Hence $X$ is a proper $D$-space.

(ii) $\rightarrow$ (iii) Let $x, y \in X$ and $U$ be a neighborhood of $\theta^{-1}(\{(x, y)\})=((x, y)) \times\{x\}$. Since $\theta^{-1}(\{(x, y)\})$ is compact, there are neighborhood $U^{\prime}$ of $((x, y))$ and $W$ of $\{x\}$ such that $U^{\prime} \times W \subseteq U$. So by (ii) there are neighborhood $V_{x}$ of $x$ and $V_{y}$ of $y$ such that $\left(\left(V_{x}, V_{y}\right)\right) \subseteq U^{\prime}$. But $\theta^{-1}\left(\left(V_{x} \cap W\right) \times V_{y}\right) \subseteq U^{\prime} \times W \subseteq U$. Hence (iii) hold.

(iii) $\rightarrow$ (ii) Let $x, y \in X$ and $U \in N((x, y))$. Then $U \times X \in N((x, y)) \times\{x\}$. Thus $U \times X \in N \theta^{-1}(x, y)$. So by (iii) there exists $V \in N(x, y)$ such that $\theta^{-1}(V) \subseteq U \times X$. Then there are neighborhoods $V_{x}$ of $x$ and $V_{y}$ of $y$ such that $\theta^{-1}\left(V_{x} \times V_{y}\right) \subseteq U \times X$. Hence (ii) holds.

Corollary 3.20. Let $X$ be a proper D-space, choose a point $x \in X$ and let $U$ be neighborhood of the stabilizer $D_{x}$ of $x$. Then $x$ has a neighborhood $V$ such that $U$ contains the stabilizer of all points in $V$.

Proof. Since $U$ is neighborhood of the stabilizer $D_{x}$ of $x, U \in N\left(D_{x}\right)$. Since $D_{x}=((x, x)), \quad U \in N(((x, x)))$. So by Proposition 3.19, there exists $V_{x} \in N(x, x)$ such that $\left(\left(V_{x}, V_{x}\right)\right) \subseteq U$. Let $y \in V_{x}$. Then $D_{y} \subseteq\left(\left(V_{x}, V_{x}\right)\right) \subseteq U$. 


\section{Palais Proper $D$-space}

From now on, the $D$-space we mean a completely regular topological $T_{2}$-space $X$ on which a locally compact and non-compact, $T_{2}$-topological $d$-algebra $D$ continuously on the left (always in the sense of Palais proper $D$-space).

Definition 4.1. Let $X$ be a $D$-space. A subset $A$ of $X$ is said to be thin relative to a subset $B$ of $X$ if the set $((A, B))=\{d \in D / d A \cap B \neq \varnothing\}$ has a neighborhood whose closure is compact in $D$. If $A$ is thin relative to itself, then it is called thin.

Remark 4.2. The thin sets have the following properties:

(i) If $A$ and $B$ are relative thin and $K_{1} \subseteq A$ and $K_{2} \subseteq B$, then $K_{1}$ and $K_{2}$ are relatively thin.

(ii) Let $X$ be a $D$-space and $K_{1}$ and $K_{2}$ be compact subsets of $X$. Then $\left(\left(K_{1}, K_{2}\right)\right)$ is closed in $D$.

(iii) If $K_{1}$ and $K_{2}$ are compact subsets of $D$-space $X$ such that $K_{1}$ and $K_{2}$ are relatively thin, then $\left(\left(K_{1}, K_{2}\right)\right)$ is a compact subset of $D$.

Definition 4.3. A subset $S$ of $D$-space $X$ is a small subset if each point of $X$ has neighborhood which is thin relative to $S$.

Theorem 4.4. Let $X$ be a D-space. Then:

(i) Each small neighborhood of a point $x$ contains a thin neighborhood of $x$.

(ii) A subset of a small set is small.

(iii) A finite union of small sets is small.

(iv) If $S$ is a small subset of $X$ and $K$ is a compact subset of $X$, then $K$ is thin relative to $S$.

Proof. (i) Let $S$ is a small neighborhood of $x$. Then there is a neighborhood $U$ of $x$ which is thin relative to $S$. Then $((U, S))$ has neighborhood whose closure is compact. Let $V=U \cap S$. Then $V$ is neighborhood of $x$ and $((V, V)) \subseteq((U, S))$, therefore $V$ is thin neighborhood of $x$.

(ii) Let $S$ be a small set and $K \subseteq S$ and let $x \in X$. Then there exists a 
neighborhood $U$ of $x$, which is thin relative to $S$. Then $((U, K)) \subseteq((U, S))$. Thus $((U, K))$ has neighborhood whose closure is compact. Then $K$ is small.

(iii) Let $\left\{S_{i}\right\}_{i=1}^{n}$ be a finite collection of small sets and $y \in X$. Then for each $i$ there is neighborhood $K_{i}$ of $y$ such that the set $\left(\left(S_{i}, K_{i}\right)\right)$ has neighborhood whose closure is compact. Then $\bigcup_{i=1}^{n}\left(\left(S_{i}, K_{i}\right)\right)$ has neighborhood whose closure is compact. But $\left(\left(\bigcup_{i=1}^{n} S_{i}, \bigcap_{i=1}^{n} K_{i}\right)\right) \subseteq \bigcup_{i=1}^{n}\left(\left(S_{i}, K_{i}\right)\right)$, thus $\bigcup_{i=1}^{n} S_{i}$ is a small set.

(iv) Let $S$ be a small set and $K$ be compact. Then there is a neighborhood $U_{k}$ of $K$, $\forall k \in K$, such that $U_{k}$ is thin relative to $S$. Since $K \subseteq \bigcup_{k \in K} U_{k}$, i.e., $\left\{U_{k}\right\}_{k \in K}$ is open cover of $K$ which is compact, so there is a finite sub cover $\left\{U_{k_{i}}\right\}_{i=i}^{n}$ of $\left\{U_{k}\right\}_{k \in K}$. Since $\left(\left(U_{k_{i}}, S\right)\right)$ has neighborhood whose closure is compact, thus $\left(\left(\bigcup_{i=1}^{n} U_{k_{i}}, S\right)\right)$ so is. But $((K, S)) \subseteq\left(\left(\bigcup_{i=1}^{n} U_{k_{i}}, S\right)\right)$, therefore $K$ is thin relative to $S$.

Definition 4.5. A $D$-space $X$ is said to be a Palais proper $D$-space if every point $x$ in $X$ has a neighborhood which is small set.

Examples 4.6. (i) Let $Z_{3}=\{-1,0,1\}$ and $*$ be defined by following table:

\begin{tabular}{|c|c|c|c|}
\hline$*$ & 0 & -1 & 1 \\
\hline 0 & 0 & 0 & 0 \\
\hline-1 & -1 & 0 & -1 \\
\hline 1 & 1 & 1 & 0 \\
\hline
\end{tabular}

\section{Table 4}

Then $Z_{3}$ act on itself (as $Z_{3}$ with discrete topology) as follows:

$$
r_{1} \cdot r_{2}=r_{1} * r_{2} \quad \forall r_{1}, r_{2} \in Z_{3}
$$

It is clear that for each point $x \in Z_{3}$, there is a neighborhood which is small $U$ of $x$, i.e., for any point $y$ of $Z_{3}$, there exists a neighborhood $V$ of $y$, then $((U, V))=$ 
$\left\{r \in Z_{3} / r U \cap V \neq \varnothing\right\}=Z_{3}$, then $((U, V))$ has neighborhood whose closure is compact.

(ii) $R$ is locally compact topological $d$-algebra (as $R$ with discrete topology and binary operation $a * b=a(a-b)$ ) acts on the completely regular Hausdorff space $R^{2}$ as follows:

$$
r \cdot\left(x_{1}, x_{2}\right)=\left(x_{1}, x_{2}\right) \text {, for every } r \in R-\{0\} \text { and }\left(x_{1}, x_{2}\right) \in R^{2} .
$$

Clear $R^{2}$ is $R$-space. But $(0,0) \in R^{2}$ has no neighborhood which is a small. Since $((U, V))=R$ for any two neighborhoods $U$ and $V$ of $(0,0)$ and since $R$ is not compact, $R^{2}$ is not a Palais proper $R$-space.

Proposition 4.7. Let $X$ be a D-space. Then:

(i) If $X$ is Palais proper D-space, then every compact subset of $X$ is a small set.

(ii) If $X$ is a Palais proper $D$-space and $K$ is a compact subset of $X$, then $((K, K))$ is a compact subset of $D$.

Proof. (i) Let $A$ be a subset of $X$ such that $A$ is compact and let $x \in X$. Since $X$ is a proper $D$-space, there is a neighborhood $U$ of $x$ which is small. Then for every $a \in A$ there exists a neighborhood $U_{a}$ which is small, then $A \subseteq \bigcup_{a \in A} U_{a}$. Since $A$ is compact, there exists $a_{1}, a_{2}, \ldots, a_{n} \in A$ such that $A \subseteq \bigcup_{i=1}^{n} U_{a_{i}}$. Thus by Theorem 4.4(ii)(iii), $A$ is a small set in $X$.

(ii) Let $X$ be a proper $D$-space and $K$ be compact. Then by (i), $K$ is a small subset of $X$, and by Theorem 4.4(iv), $K$ is thin. So $((K, K))$ has neighborhood whose closure is compact. Then by Remark 4.2(iii), $((K, K))$ is closed in $D$. Thus $((K, K))$ is compact.

Proposition 4.8. Let $X$ be a $D$-space and $y$ be a point in $X$. Then $y$ has no small set whenever $y \in J(x)$ for some point $x \in X$.

Proof. Let $y \in J(x)$. Then there is a net $\left(d_{g}\right)_{g \in G}$ in $D$ with $d_{g} \rightarrow \infty$ and a net $\left(\chi_{g}\right)_{g \in G}$ in $X$ with $\chi_{g} \rightarrow x$ such that $d_{g} \chi_{g} \rightarrow y$. Now, for each neighborhood $S$ of $y$ 
and every neighborhood $U$ of $x$ there is $g_{0} \in G$ such that $\chi_{g} \in U$ and $d_{g} \chi_{g} \in S$ for each $g \geq g_{0}$, thus $d_{g} \in((U, S))$, but $d_{g} \rightarrow \infty$, thus $((U, S))$ has no compact closure, i.e., $S$ is not a small neighborhood.

Proposition 4.9. Let $X$ be a Palais proper D-space. Then $J(x)=\varnothing$ for each $x \in X$.

Proof. Suppose that there exists $x \in X$ such that $J(x) \neq \varnothing$, then there exists $y \in J(x)$. Thus there is a net $\left(d_{g}\right)_{g \in G}$ in $D$ with $d_{g} \rightarrow \infty$ and a net $\left(\chi_{g}\right)_{g \in G}$ in $X$ with $\chi_{g} \rightarrow x$ such that $d_{g} \chi_{g} \rightarrow y$. Since $X$ be a proper $D$-space, there is a small (thin) neighborhood $U$ of $x$. Thus, there is $g_{0} \in G$ such that $d_{g} \chi_{g} \in U$ and $\chi_{g} \in U$ for each $g \geq g_{0}$, so $d_{g} \in((U, U))$, which has a compact closure. Therefore $\left(d_{g}\right)_{g \in G}$ must have a convergent subnet, which is a contradiction. Thus $J(x)=\varnothing$ for each $x \in X$.

Proposition 4.10 [3]. Let $X$ be a periodic D-space. Then $J(x)=\varnothing$ for each $x \in X$ if and only if every pair of points of $X$ such that $D x \neq D y$ has relatively thin neighborhood.

Proposition 4.11. Let $X$ be a periodic D-space and $D x \neq D y$ for every pair of points $x, y$ of $X$. Then the definition of Palais proper D-space and the definition Bourbaki proper D-space are equivalent.

Proof. $(\Rightarrow)$ By Propositions 4.10 and 3.17.

$(\Leftarrow)$ Let $X$ be a Bourbaki proper $D$-space. Then by Proposition 3.17, $J(x)=\varnothing$ for each $x \in X$. Let $x \in X$. We will show that $x$ has a small neighborhood. By Proposition 4.10 , there a small neighborhood $U_{x}$ of $x$. Thus $X$ is Palais proper $D$-space.

\section{References}

[1] N. Bourbaki, General topology, Chapter 1-4, Elements of Mathematics, 2nd ed., SpringerVerlag, Heidelberg, New York, Pairs, Tokyo, 1989.

[2] K. Habeeb and T. Ahmed, Topological transformation $d$-algebra, The 23rd Specialized Scientific Conference of the Faculty of Education at University of Mustansiriya, 26-27 April 2017, Vol. 1, 2017, pp. 456-464. 
[3] H. Kareem Abdulla, H. Jebur Ali and A. Talip Hussein, Limit sets and Cartan D-space, Journal of Al-Qadisiyah for Computer Science and Mathematics 9(2) (2017), 134-140. https://doi.org/10.29304/jqcm.2017.9.2.305

[4] Q. P. Hu and X. Li, On BCH-algebras, Math. Sem. Notes Kobe Univ. 11 (1983), 313-320.

[5] Q. P. Hu and X. Li, On proper BCH-algebras, Math. Japon. 30 (1985), 659-661.

[6] Y. Imai and K. Iséki, On axiom systems of propositional calculi XIV, Proc. Japan Acad. Ser. A Math. Sci. 42 (1966), 19-22. https://doi.org/10.3792/pja/1195522169

[7] K. Iséki, An algebra related with a propositional calculus, Proc. Japan Acad. Ser. A Math. Sci. 42 (1966), 26-29. https://doi.org/10.3792/pja/1195522171

[8] J. Neggers, Young Bae Jun and Hee Sik Kim, On $d$-ideals in $d$-algebras, Math. Slovaca 49(3) (1999), 243-251.

[9] J. Neggers and H. S. Kim, On $d$-algebras, Math. Slovaca 49 (1999), 19-26.

[10] T. Ahmed, K. Habeeb and J. Haider, Uniformity in $d$-algebras, Earthline J. Math. Sci. 2(1) (2019), 207-221. https://doi.org/10.34198/ejms.2119.207221 